VII.

\title{
Die physiologisehe Bedeutung des Ammoniaks.
}

\author{
Von \\ Pietro Albertoni, Bologna. \\ (Deutsche Übertragung von Frau Else A sher.) \\ Literatur.
}

1. A bderbalden E., Fütterungsversuche mit vollständig bis zn Aminosäuren abgebautem Eiweiss und mit Ammonsalzen. Zeitschr. f. phys. Chemie. Bd. 78. p. 1. 1912.

2. Derselbe und P. Hirsch, Fütterungsversuche mit Gelatine, Ammonsalzen, vollständig abgebauten Fleisch an jungen Hunden. Zeitschr. f. phys. Chemie. Bd. 81. p. 323. 1912.

3. Derselbe, Weitere Studien über den Stickstoffwechsel. Zeitschr. f. phys. Chemie. Bd. 96. p. 29. 1915/16.

4. Albertoni P., Recherches sur les modifications du sang consécutives à l'extirpation de l'appareil thyro-parathyroiden. Arch. Intern. d. Phys. Vol. XI. p. 29. 1911. Istituto fisiologico di Bologna.

5. Beaunis-A ducco, Vol. 1. p. 474-475 e Liotta v. N. 47.

6. Beccari L., Sul dosamento dell' ammoniaca del sangue. Bull. delle scienze mediche. Bologna. 1905. p. 292. Istituto fisiologico di Bologna.

7. Derselbe, Sulla eliminazione dell' ammoniaca nei grossi erbivori. Mem. d. R. Accademia di Scienze di Bologna. Serie VII. Tomo III. p. 253. 1916. Istituto fisiologico di Bologna.

8. Derselbe, Sul comportamento dell' ammoniaca nell' organismo. Mem. d. R. Accademia d. Scienze d. Bologna. Serie VII. Tomo IV. p. 213. 1917. Istituto fisiologico d. Bologna.

9. Biedl und Winterberg, Beiträge zur Lehre von der ammoniakentgiftenden Funktion der Leber. Pflügers Arch. Bd. 88. p. 140. 1902.

10. Björn-Andersen und Laúritzen, Über Süure- und Ammoniakbestimmung im Urin und ihre klinische Anwendung. Zeitschr. f. phys. Chemie. Bd. 64. p. 21. 1910.

11. Camerer W. jun., Beobachtungen und Versuche über die Ammoniakausscheidung im menschlichen Urin, mit Berücksichtigung noch weiterer stickstofihaltigen Bestandteile und Bestimmung der Azidität nach Li eblein. Zeitsehr. f. Biologie. Bd. 43. p. 13. 1902.

12. Carlson und Jacobson Clara, The depression of the ammonia destroying power of the lever after complete thyroidectomy. Americ. Journ. of Pbys. Vol. 25. p. 403. 1910 and Vol. 28. p. 13. 1911.

13. Denis N. and Minot A. S., Ammonia excretion as influenced by the ingestion of alkalis. Journ. Biol, Chem. Vol. 35. p. 108. 1918.

14. Embden und Schmitz, Uber synthetische Bildung von Aminosïuren in der Leber Biochem. Zeitschr. Bd. 29. p. 423. 1910. 
15. Eppinger, Zeitschr. f. expr. Path. u. Ther. Bd. 3. p. 530.1906.

16. Folin 0., Eine neue Methode zur Bestimmung des Ammoniaks im Harn und anderen tierischen Flüssigkeiten. Zeitschr. f. phys. Chemie. Bd. 27. p. 161.1902.

17. Derselbe, Estimation of ammonia in blood. Journ. Biol, Chem. Vol. 39. p. 259. 1919.

18. Derselbe und Denis, The origin and significance of the ammonia in portal blood Journ. of biol. Chem. Vol. II. p. 161. 1912.

19. Dieselben, Nitrogen determinations by direct neutralization. Journ. Biol. Chem. Vol. 26. p. 497. 1916.

20. Gad-A ndersen, Concentration of ammonia in different secretions and tissues. Journ. Biol. Chem. Vol. 39. p. 267. 1919.

21. Gammaltoft S. A., Über die Ammoniakmenge im Harn und deren Verhälnisse zur Ventrikelsekretion. Zeitschr. f. phys. Chemie. Bd. 75. p. 57. 1911.

22. Gerard, Dosage de $\mathrm{l}^{\prime} \mathrm{NH}_{3}$ du sang par une méthode volumetrique. Compt. rend. Soc. Biol. p. 1186. 1919.

23. Gessler Hans, Zur Frage des Wesens der Stickstoffretention bei Fütterung mit Ammoniaksalzen. Zeitschr. f. phys. Chem. Ba. 109. p. 280.1920.

24. Grafe E., Zur Frage der Stickstoffretentionen bei Fütterung von Ammoniumchlorid. Zeitschr. f. phys. Chemie. Bd. 90. p. 75. 1914.

25. Derselbe, Über Stickstoffersparnis durch Darreichung von Ammoniaksalzen und Harnstoff bei Menschen. Deutsch. Arch. f. klin. Med. Bd. 117. p. 448. 1916.

26. Derselbe und Schläpfer, Öber Stickstoffretentionen und Stickstoffgleichgewicht bei Fütterung von Ammoniaksalzen. Zeitschr. f. phys. Chemie. Bd. 77. p. 1. 1912.

27. Derselbe und Wintz, Über die Beeinflussung des Stickstoffwechsels durch Fütterung von Natriumnitrat. Zeitschr. f. phys. Chem. Bd. 86. p. 283.1914.

28. Hahn A. und Kootz E., Quantitative Bestimmung des Ammoniaks im Crin, in serösen Flüssigkeiten usw. Bioch. Zeitschr. Bd. 105. p. 220, 1920.

29. Haskins H. D., Nitrogenous metabolimas affected by diet and alkaline diuretic. Journ. of biol. Chem. Vol. 2. p. 217. 1906.

30. Has selbach K. A., Ammoniak als physiologischer Neutralitätsregulator. Bioch. Zeitschr. Bd. 74. p. 18. 1917.

31. Henderson L. J., A critical study of the process of acid excretion. Journ. of biol. Chem. Vol. 9. p. 403. 1911.

32. Derselbe and Palmer, On the intensity of urinary acidity in normal and pathological conditions. Journ. of biol. Chem. Vol. 13. p. 393. 1912.

33. Dieselben, On the several factors of acid excretion. Journ. of biol. Chem. Vol. 17. p. 305. 1914.

34. Dieselben, On the several factors of acid excretion in nephritis. Journ. of biol. Chem. Vol. 21. p. 37. 1915.

35. Henrique $z$ und Andersen, Über die Stickstoffretentionen bei Zufuhr von Ammoniaksalzen oder Harnstoff. Zeitschr. f. phys. Chem. Bd. 92. p. 21. 1914.

36. Derselbe und Christiansen E., Untersuchungen über die Ammoniakmenge im Blute. Biochem. Zeitschr. Bd. 78. p. 165. 1916 u. Bd. 80. p. 297. 1917.

37. Höber R., lat die Lunge für Ammoniak durchgängig? Pflügers Archn Bd. 149. p. 87. 1913.

38. Hopkins and Denis, Interrelation of the Ammonia and carbon dioxyd content of the blood. Jomrn. of biol. Chem. Vol. 10. p. 407. 1911.

39. Horodynski, Salaskin, Zaleski, Über die Verteilung des Ammoniaks im Blute und den Organen normaler and hungernder Hunde. Zeitschr. f. phys. Chem. Bd. 35. p. 245.1902.

40. Jacobi, Über die Harnstoffbildung im Organismus. Erg. d. Physiol. T. p. 532. 1902.

41. Janney N., Die Ammoniakausscheidung im menschlichen Harne bei Zufuhr von Harnstoff und Natron. Zeitschr. f. phys. Chem. Bd. 76. p. 99. 1912.

42. Kn o op F, Über den physiologischen Abbau der Säuren und aie Synthese einer Aminasüure im Tierkörper. Zeitschr. f. physiol. Chem. Bd. 67. p. 489. 1910. 
43. Kowalewski und Salaskin, Über den Ammoniak. und Milehsiuregehalt im Blut und uber die stickstoffrerteilung im Haru von Hasen unter verschiedenen Verhaltnissen. Zeitschr. p. phys. Chem. Bd. 35. p. 552. 1902.

44. Derselbe und Markewicz, Oher das Sehicksal des Ammoniaks im Organismns des Hundes bei intravenöser Injektion von Kohlensãuren-Ammoniak. Biochem. Zeitschr. Bd. 4. p. 196. 1907 .

45. Lafayette B., Mendel and Rose W. C., Journ. of hiol. Chem. Vol. 10. p. 217. 191112.

46. Leclère A., Déterminations de l'ammoniaque dans l'urine. Compt. rend. Soc. Bisl. Vol. 80. p. 959. 1917.

47. Liotta D., L'eliminazione dell'ammoniara con l'mina durante il riposn. Arela. d. Firmacol sper. e Scienze affini Vol. 22. p. 20is. 1916.

48. Marf o r i P., Uber die Ammoniakmengen, welche der Organismus in Larnstoft umzuwandeln vermag. Schmiedebergs Arch. Bd. 33. p. 71. 1892.

49. Moritz F., Uber die Bestimmung der Bilanz von Särren und Basen in tierischen Fliissig keiten. Deutseh. Arch. f. klin. Med. Bd. 83/84. 1905.

50. Moscati G, L'ammoniaca dell'urinat nelle varie diete. 1911.

51. Derselbe, Linfluenza del riposo e del movimento sull'eliminazione dellammoniara coll'urina 1911.

52. Nencki tet 7aleski, Du dosage de l'ammoniaque dans les sues et les organes dés animaux. Arch. de Sc. biol. de St. Petersbourg. Vol. 9. p. 232. 1901.

53. Peschek H., Pfügers Arch. Bd. 142. p. 143. 1911 enthalt die Literatur uber diese orste Periode.

54. Derselbe, Über alie Finwirknng von Ammoniaksalzen und essigsauren Salzen ant den Stoftwechsel ter Fleischfresser. Biochem. Keitschr. Bd. 45. p. 244. 1912.

j5. Derselhe, Weitere Versuche am Fleischfresser über die stickstoffsparende Wirkung von Saizen, besonders von Natriumazetat. Biochem. Zeitschr. Bd. 52. p. 275.1913.

56. Dersel be, Weitere Versuche aber die stickstoffersparende Wirkung von Natriumazetat. beim Wiederkäuer. Biochem. Zeitschr. Bd, 62. p. 186. 1914.

57. Piceinini $G$., Ta diffusione dell'ammoniaca neliorganismo in rapporto con l'intossicazione e l'autointossicazione per detta sostanza. Bull. delle Scienze Mediche Bologna. 190 . p. 31. Istituto fisiologico di Bologna.

58. Derselbe, Lammoniaca nellaria espirata o nel sangne. Atch. di farmuc a scienze aftini Vol. 5. p. 36. 1906.

59. Pincuss ohn L., Über Ammoniakbestimmang im Harn. Bioch. Zeitschr. Bd. 99. p. 267. 1919.

60. Salk ow ski E., Zur Kenntnis der Eiweỉsskörper der Füulnisbakterien. Zeitschr. f. phys. Chem. Bd. 109. p. 49.1920.

61. Derselbe, Zur Kenntniss der Fiweissbildung aus Harnstofl bei Wiederkunnen. Zeitschr. 1. phys. Chem. Bd. 109. p. 276. 1920.

62. Schenitzky Ch., Zur Nethodik der Ammoniakbestimmung des menschlichen Iarnes: vergleichende Bestimmung mit den Apparaten Schlösing, Krüger, Reich., Schittenlelms und Hahns. Biol. Zeitschr. Bd. 76. p. 177. 1916.

63. Schittenhelm und Katzenstein, Ober die Beziehungen des Ammoniaks zum Gesamtstickstoff im Urin. Fin Betrag zur Azidose. Zeitschr. f. exper. Path, und Ther. Bd. ${ }^{\prime \prime}$. p. 542.1905.

64. Shermann H. C. and Gettier A. O., The balance of acid-forming and hase-forming elements in foods, and its relation to ammonia metabolism. Journ. bioch. Ghem. Vol, 11. p. 323.1912.

64a. Spiro, K., Zur Methodik der Ammoniak- und Harnstoffbestimmungen im Harn. Hoime isters Beitr. z. chem. Pliys. u. Path. 1907. Bd. 9. S. 481.

65. Summer J. B., The direct determination of urea and ammonia in muscle. Jonrn. Binl. Chem. Vol. 26. p. 95. 1916.

66. Derselbe, Direct nentralization of Ammonia in urin. Jonrn. Hiol. Chem. Vol. 34. p. 37. 1918. 
67. Taylor A. E. and Ringer A, J., The utilisation of ammonia in the protein metabolism. Journ. of. biol. Chem. Vol. 14. p. 407. 1913.

68. T'ullio $\mathrm{P}$., Sul contenuto in ammoniaca del sangue delluomo durante il riposo e il lavoro muscolare. Arch. di fisiologia. Vol. 10. p. 71. 1912. Jstituto fisiologico di Bologina.

69. Underhill F. P., Studies on the metabolism of Ammoniumsalts. 1. Journ. Biol. Chem. Vol. 15. p. 3271913.

70. Derselbe, Studies on the metabolism of Ammoniumsalts. II. Journ. Biol. Chem. Vol. 15. p. 337. 1913.

71. Derselbe and Saiki, The iufluence of complete thyroidectomy and of thyroid feeding upon certain phases of intermediary melabolism. Journ. of. biol. Chem. Vol, 5. p. 226. 1908.

72. Derselbe and Goldschmidt, Studies on the metabolism. of Ammoniumsalts. Journ. of biol. Chem. Vol. 15. p. 337 u. 341.1913.

73. Dersel be and Bogert T. L., Alterations in the outpat of certain urinary constituent as determined by changes in the character of the diet. Journ. Biol. Chem. Vol. 27. p. 161. 1916.

74. Wilson, Stearus and Ianuey, The excretion of atids and ammonia after parathyroideclony, Journ. of biol. Chem. Vol. 23. p. 123. 1915

Die Haupttatsachen über den Stofïwechsel und über die physiologische Bedeutung des Ammoniaks, welche bis zum Jahre 1900 bekannt waren, sind in diesen Ergebnissen von Jacobi (40) in dem Artikel, auf welchen ich für die frühere Literatur hinweise, dargestellt worden.

Die Untersuchungen über den Stoffwechsel des Ammoniaks im tierischen Organismus erhielten einen veuen Impuls und eine strengere Abgrenzung, seitdem Nencki und Zaleski (52) ihre Methode der Bestimmung einfübrten, welche auf dem Prinzip der Destillation im Vakuum begründet ist, und welche dadurch, dass sie verschiedene Fehlerquellen beseitigte, gestattete, das im Blute, in den Organen und in den verschiedenen Körperflüssigkeiten befindliche Ammoniak genau zu bestimmen.

Ausser diesen seither berühmten Versuchen von $\mathrm{Neacki}$ und $\mathrm{Pawlow}$ über das Verhalten des Ammoniaks bei operierten Tieren mit Eckscher Fistel und über die Schutzwirkung der Leber, fand diese Methode noch andere zahlreiche und interessante Anwendungen.

Bald danach beschrieb $O$. Folin (16) eine Methode zur Ammoniakbestimmung, welche auf dem Prinzip gegründet war, vermittelst eines andauernden Luftstromes das Ammoniak zu entfernen, eine Methode, welche, weil sie leichter zu benutzen war, vielfach angewendet wurde und bald zahlreiche Modifikationen erhielt, die zu beschreiben hiei nieht der Ort ist (Shaffer, spiro, Krüger und Reich, Malfatti, Wolff u. a).

Eine Modifikation der Methode von Nencki und Zaleski, die immer die genaueste bleibt, ist von Beccari.(16) ersonnen worden, durch welche er das Verfahren leichter und sicherer gestaltet hat. Un die Unannehmlichkeiten zu vermeiden, welche durch den reichlichen Schaum entstehen, der sich beim 
Destillieren von eiweisshaltigen Flüssigkeiten, wie dom Blute, bildet, hat Beccar i die Form des Destillierapparates modifiziert, indem er das Blut nach und nach durch ein Glasröbrchen, fast einer Kapillare, in den Destillierkolben gelangen liess. Derselbe ist ein Glaskolben, Typus Ladenburg, mit drei Marken mit einer Kapazität von $500-700 \mathrm{cem}$. Nachdem man vermittelst einer Wasserpumpe den Raum luftleer gemacht hat, lässt man das Blut durch die Kapillarröhre eintreten, wobei man den Zufluss durch eine Schraubenklemme reguliert, welche einen Gummischlauch komprimiert, der sich zwischen der Kapillare selbst und dem Rezipienten, welcher das zu analysierende Blui enthält, verbindet. Die Bluttröpfchen bilden allmählich, wenn sie das äussere Ende der Kapillare, die sich mitten im Glaskolben befindet, erreichen, Bläschen, welche bald platzen, wobei sie die Wände des Gefässes, welches in ein Wasserbad von $40^{\circ}$ eingetaucht ist, bespritzen und bald verdampfen.

Das Ammoniak wird durch die Alkalinität des Blutes, durch die Wirkung des niedrigen Druckes und der Temperatur ausgetrieben. Diese Methode ist auch sehr gut für die Untersuchung des Harns, da sie die schweren Fehlerquellen des Verfahrens von Salkowski ausschliesst und die Untersuchungen viel rascher und genauer gestaitet.

Die Resultate, welche man mit diesem Verfahren erhält, sind sichor und genau, auch bei schwierigeren Untersuchungen. Die gefundenen Werte bei arteriellem Blute des Hundes sind etwas höher als die von Nencki und Saleski augegebenen, aber das rührt gerade von der vollständigeren Destillation des Blutes her; die Daten von B e c car i sind durch neuere Versuche, welche in diesem physiologischen Institut von Piccinini u. a. ausgeführt wurden, bestätigt worden.

Piccinini (57) hat auch dasselbe Verfahren zur Bestimmung des Ammoniaks in den Geweben eingeschlagen, wobei er die Methode von Beccari für das Blut und den Harn leicht modifizierte. Er erhielt ausgezeichnete Resultate, wodureh er einen bemerkenswerten Beitrag zur genaueren Kenntnis der Diffusion des Ammoniaks in den Organen, sowoh? unter normalen wio unter besonderen experimentellen Bedingungen, geliefert hat.

Mit der ursprünglichen Methode von Nencki wurde diese grundlegende Tatsache für den Stoffwechsel des Ammoniaks in weitem Umfange von Horodynski, Salaskin und Zaleski (39) bearbeitet, wobei schon im Jahre 1902 die ursprünglichen Angaben von Nencki und Zaleski über den Ammoniakgehalt des arteriellen Blutes, über die Zunahme des Ammoniaks in der Vena Porta und über die Funktion der Leber diese Substanz in Harnstoff zu verwandeln, bestätigt wurden. Auch das in den Organen enthaltene Ammoniak ist ziemlich konstant und beim Hunger nimmt es, ausser im Gehirn, zu.

Im Gegensatz zu dem, was diese verschiedenen Forscher gefunden haben, hat Gad-Andersen (20) kürzlich beobachtet, dass die Konzeutration des 
Ammoniaks sowohl im Blute wie in den Muskelu dieselbe ist; dasselbe ist der Fall im Herzen, in der Leber, im Fett, in der Galle, in der Zerebrospinalflüssigkeit, im Humor aq. Nach dem Tode der Gewebe bildet sich Ammoniak aul Kosten des Harnstoffes.

Wie wie bereits erwähnt haben, entstehen, hiervon ausgehend, eine grosse Anzahl von Untersuchungen über das Ammoniak im Organismus. Diese Untersuchungen bewegten sich in verschiedenen Richtungen mit dem Zwecke die verschiedenen Probleme aufzuklären, welche sich auf dessen Entstehung und auf seine Funktion bei seinen Umwandlungen beziehen.

Daher sind in bezug auf die Technik viele andere Arbeiten veröffentlicht worden, welche zu behandeln hier nicht der Ort ist. Wir wollen von den wichtigsten diejenigen von Folin und Denis (19), Schnetzky (62), Summer (65, 66), Leclère (46), Folin (17), Gérard (25), Pincussohn (59), Hahn und Kootz (28), erwäluen.

Über den Ammoniakgehalt des Blutes in den verschiedenen Regionen bestatigen die Untersuchungen von Henriquez und Christiansen (36) in der Mehrzahl die schon bekannten Daten; die berichteten Mittelwerte derselben sind etwas niedriger als diejenigen, welche man bei der Destillation im Vakuum erhält; aber diese Methode ist sicherer und immer jener vorzuziehen, wegen der schon von Folin vorgeschlagenen und von den erwähnten Forschern angewandten Entfernung des Ammoniaks. Diese haben auch gefunden, dass die Blutkörperchen mehr Ammoniak enthalten als das Plasma.

Bei den Veränderungen, welche das Ammoniak im Organismus erleiden kann, ist an die Arbeiten von Biedl und Winterberg (9), von Kowalewsky und Markewicz (44), und von Underhill (69) zu erinnern, welche untersuchten wie sich die in das Tier eingeführten Ammonsalze zur gallenbildenden Funktion der Leber, zur Anhäufung derselben in den verschiedenen Organen und ibrer Ausscheidung verhalten. Derartige Untersuchungen schliessen sich an die früheren von Marfori (48) über die Menge von Ammoniak, welche der Organismus in Harnstoff verwandeln kann, an.

Andere Untersuchungen haben sich dem Studium der Modifikationen zugewandt, welche durch verschiedene physiologische Prozesse auf den Ammoniakstoffwechsel verursacht werden. So hat P. Tullio (68) den Einfluss der Muskelarbeit auf den Ammoniakgehalt des Blutes untersucht, wobei er zu dem Schlusse kam, dass beim Menschen der Muskel während der Arbeit in einer Zeiteinheit eine grössere Menge von Ammoniak erzeugt und ausscheidet als im Ruhestand.

Hopkins und Den is (38) haben Beziehungen zwischen der Kohlensäure und dem Ammoniak des Blutes untersucht und fanden, dass, während bei hungernden Hunden die Asphyxie eine Verminderung des kreisenden Ammoniaks erzeugt, bei Tieren in voller Verdauung im Gegenteil diese Beziehung umgekehrt ist, 
Folin und Denis (18), welche die Entstehung des Ammoniaks im Portaderblut untersuchten, haben durch Versuche an Katzen gezeigt, dass während der Darmverdauung das Blut, welches aus dem Dickdarm kommt, reicher an Ammoniak ist als dasjenige, welches aus dem Düundarm kommt und schlossen daraus, dass die Zunahme von Ammoniak, die sich im Blute der Vena Porta offenbart, grossenteils von dem im Dickdarm gebildeten Ammoniak stammt. Gammelt oft (21) hat hingegen die Bezielsungen untersucht, welche zwischen der Absonderung der Salzsäure im Magen und der Ausscheidung des Ammoniaks im Harn existieren, in dem er seine Resultate auf Grund der Modifikationen der Alkalinität des Blutes, welche mit beiden Vorgängen in Zusammenhang stehen, erklärte.

Über die Ausscheidungswege des Ammoniaks sind, ausser zahlreichen Versuchen über die Harnausscheidung, von welcher wir sogleich sprechen werden, die Arbeiten von Picciniui (58) und von $\mathrm{Höber}(57)$ über die strittige Frage der Ausscheidung von Ammoniak in der Ausatmungsluft zu erwälmen. Ersterer hat mit genauen Versuchen zweifelsohne festgestellt, dass es keine Ausscheidung von Ammoniak bei der Atmung gibt. Letzterer hat gezeigt, dass dies nicht von der Undurchlässigkeit der Lunge für diese Substanz herrührt, sondern von der grossen Löslichkeit und der Verbindung des Ammouiaks im Blute selbst, so dass man, wenn man ein lier Luft einatmen lässt, die eine bestimmte Ammoniakmenge enthält, zeigen kann, dass dieselbo ins Blut übertritt.

Die Untersuchungen über den Ammoniakstoftwechsel haben auch einen physio-pathologischen Zweck verfolgt, mit der Absicht die Beziehungen derselben zu krankhaften Zuständen, vor allem zu solchen mit giftigem Charakter aufzuklären. Wir können hier die Arbeiten von Piccinini (57) über die experimentelle Urämie und diejenigen von Henders on und Palmer (34) über die Ausscheidung von Ammoniak im Harn bei Nierenkranken erwähnen.

Carlson und Clara Jacobson (12) haben behauptet, dass Ammoniak im Blute von Tieren zunimmt, denen man die Schilddrüso und die Nebenschilddrüse entfernt hat, und dass die l'etanieanfälle von einer Anhäufung von Ammoniak herrühren. Aber A l bert on i hatte schon früher widersprechende Resultate erhalten und er hat in einer neuen Versuchsreihe (4) mit der Methode von Beccari beim hungernden Huude 0,63 mg Ammoniak aul 100 Blut gefunden und nach der Exstirpation des thyreo-parathyreotischen Apparates während Tetanieanfällen 0,60 und $0,34 \mathrm{mg}$ auf 100 , d. h. keinerlei Zunahme gegenüber den normalen Zahlen. Übrigens braucht die leichte Zunahme, welche Carlson und Clara Jacobson im Blute gefunden haben, nicht die Ursache Eür tetanische Anfälle zu sein, sie kann durch den Hunger, die Krä̀mpfe, welche die Acidosis verursachen, hervorgerulen worden sein. Sic kann nämlich eine indirekte Folge der Vernichtung der Schilddrüsenfunktion seiu. Es kommt noch hinzu, dass die Funktionen der Teber mehr oder weniger beeinträchtigt 
werden und daher auch die synthetische Bildung von Harnstoff herabgesetzt wird.

In diesem Sinne müssen die Beobachtungen von Un de rhill und Sa i ki (71) gedeutet werden, welche nach der totalen Thyreoidektomie beim Hunde eine Zunahme von Ammoniak im Harn gefunden haben, wie auch Wilson, Stearns und Janney (74), die infolge der Parathyreoidektomie anfangs einer Verminderung der Acidität und des Ammoniaks im Harn begegnet sind, später mit der Entwickelung der Tetanio Zunahme der einen sowie des anderen.

Aber das Gebiet, auf welchem die Untersuchungen über das Ammoniak im Organismus die grössten Veränderungen herbeigeführt haben, ist dasjenige, welches die Beziehungen des Ammoniaks selbst zur Säureausscheidung und zur Alkaleszens des Blutes betrifft. Nächst der Erforschung des Ursprungs und der Umwandlung des Ammoniaks beim organischen Stickstofiwechsel ist es dieses Problem, in welchem sich am ausgesprochensten die biologische Funktion jener Substanz offenbart.

Seitdem Walter (1877) gezeigt hatte, dass die Darreichung von Säuren die Ausscheidung von Ammoniak durch den Harn bein Hunde steigert und Schmiedeberg seine Lehre über die Schutzwirkung des Ammoniaks selbst gegen Säurevergiftung entwickelt hatte, schloss man, dass die Ausscheidung dieses letzteren der sicherste Massstab für die Acidosis des Organismus sei.

Aber kurze Zeit darauf brachte die Entdeckung der Bildung des Harnstoffs aus den Ammoniakbestandteilen im Organismus (Leber) einen neven Gesichtspunkt, um die genaue physiologische Bedeutung des Ammoniaks festzusiellen. Die Modifikation des harnstoffbildenden Systems könmen einon nicht zu vernachlässigenden Teil der Ursachen ausmachen, welche die Ammoniak. ausscheidung im Harn verändern.

Salkowski hatte jedoch beobachtet, dass die Pflanzenfresser sich anders wie die Fleischfresser sowohl in bezug anf die Einführung von Säuren sowie auf die Umwandlung der verschiedenen Ammonsalze im Harnstoff verhalten; oin solcher Unterschied wurde zum 'Teil der Verschiedenheit des Nahrungsregimes zugeschrieben und der Überreichlichkeit der fixen Alkalien, welche in Organismus der Pflanzenfresser kreisen. Aber selbst wemn man einen Unterschied zwischen den einen und den abderen in der Fähigkeit Säuren vermittelst Ammoniak zu neutralisieren zugesteht, so ist doch beobachtet worden, dass bei den Herbivoren die Fähigkeit hierbei mangelhaft ist, weil die eingeführten Mineralsäuren durch die fixen Alkalien gesättigt werden und weil die Einführung solcher Säuren bald zu schweren Störungen bei diesen Tieren führt.

Diese irrige Auffassung entstand infolge einer unvollkommenen Kenntuis der Neutralitätsmechanismen der Säureradikale des Organismus vermittelst des Ammoniaks. Sie sollte jedoch nicht mehr in modernen Abhandlungen 
und Handbüchern gefunden werden, aus welchen sie dann auch in Spezialarbeiten über den Gegenstand (5) zitiert werden.

Später haben Winterberg (1898) und danach Eppinger (1906) beim Kauinchen die Fähigkeit entdeckt, Dank des Ammoniaks die eingeführten Säuren zu neutralisieren, vorausgesetzt, dass sich das Tier unter besonderen Bedingungen des Stickstoffregimes befindet.

Viele Forscher haben sich mit diesem Problem beschäftigt, sei es auf dom experimentellen Gebiet, sei es auf jenem der klinischen Beobachtung. In der Periode, welche der hier betrachteten voranging, wurde festgestellt, dass sowohl beim Hunde (Salkowski und I. Munk) wie beim Menschen (Hallervorden, Coranda) die Ammoniakausscheidung zunimmt bei Einführung von Süuren, und abnimmt bei einer solchen von Alkalien. Die zahlreichen früheren Untersuchungen tendieren, ausser dass sie in den ver. schicdensten Fällen dies allgemeine Gesetz bestätigen, hauptsächlich die Bedingungen jenes Phänomens zu präzisieren und in den feinen Mechanismus, der es regelt, einzudringen. Wir wollen die wichtigsten Arbeiten hier zitieren: Kowalewsky und Salaskin (43) untersuchten bei Gänsen die Veränderungen des Ammoniaks und der Reaktionen des Blutes und sahen, dass bei ziemlich hohen Dosen von Säuren die alkalische Reaktion abnimınt, während das Ammoniak zunimmt; es bleibeu beide bei Einführung von Alkalien unverändert. Hingegen nimmt die Ammoniakausscheidung im Harn ab bei Einführung von Alkali und zu bei Säuren.

Camerer (II) untersuchte beim Menschen das relative Verhalten der Acidität (ausgedrückt in Werten der Phosphorsäuren) und des Ammoniaks des Harns und sah, dass die Einführung von Säuren zwar die relative Ausscheidung des Ammoniaks steigert, aber ziemlich wenig die absolute, und er konnte keinen Einfluss der Alkalien auf jene Ausscheidung nachweisen.

Hingegen konnte Haskins (29) beim Menschen nachweisen, dass die Alkalien das Ammoniak des. Harns von $0,8 \mathrm{~g}$ auf $0,115 \mathrm{~g}$ in 24 Stunden herabsetzen können. Dasselbe ist auch schon von N. Jauney (41) bestätigt worden, ebenso von $\mathrm{Denis}$ und $\mathrm{M}$ inot (13), welche dieselben Tatsachen bei Nephritikern konstatierteli. Analoge Untersuchungen machten auch Moritz (49) und Schittenhelm (63). Auch Björn Andersen und Lauritzen (10) bestätigten den Parallelismus zwischen der Gesamtacidität des Harnes und der Ammoniakausscheidung sowohl in normalen wie in pathologischen Fällen, während sie zeigten, dass der Quotient des Ammoniaks einen vollkommen unabhängigen Verlauf hat und in direkter Beziehung zum Eiweissstoffwechsel steht.

Die letzte Bestätigung der engsten Beziehung zwischen der Acidosis und der Zunahme des Ammoniaks im Harn ist vou $\mathrm{Hasselbach}(30)$ geliefert worden.

Über dic Grenzen der Neutralisation, welche vom Ammoniak erreicht 
werden köunen, hat Eppinger (15) festgestellt, dass ein Hund mit Fleischdiät einer Dosis von Salzsäure widerstelien kann, die $0,9 \mathrm{~g}$ pro $\mathrm{kg}$ entspricht, da $1,80 \%$ jener Säure mit Ammoniak neutralisiert werden.

Über die Ausscheidung von Ammoniak beim Hunger gibt es sich widersprechende Angaben.

Während Brugsch (1905), Cathcart (1907) und Gräfel (1910) bei den Karnivoren und Omnivoren eine Zunahme fanden, haben L a fa y et te B. Men del und Rose (45) beim Kaninchen eine Abnahme, sci es absolut oder relativ, im ausgeschiedenen Ammoniak gesehen, welche sie der Tatsache zuschreiben, dass möglicherweise die Pflanzenfresser weniger empfänglich für die Acidosis sind, oder auch mit anderen Alkalien die Säuren neutralisieren können. Über den Einfluss der Nahrung auf die Ammoniakausscheidung besitzen wir die interessante Untersuchung von Sherman und Gettler (64) über die Fähigkeit der verschiedenen Nahrungsmittel dem Organismus Säuren oder Alkalien zu liefern, woraus folgt, dass sich das Ammoniak in bezug auf jene Eigenschaften der Nahrungsmittel verschieden verhält. Demzufolge baben diese Forscher festgestellt, dass die Zunabme des Ammoniaks nur ein Drittel oder ein Viertel der von der Nahrung erzeugten Gesamtsäure neutralisieren kann.

Ähnliche Resultate haben Underhill und Bogert (73) erhalten. Dic Ammoniakausscheidung in Beziehung $z u$ den verschiedenen Diäten wurde auch von Moseati (51) und von Liotta (47) untersucht.

Derjenige Forscher, welcher die Anregung gegeben hat, tiefer in den regulatorischen Mechanismus der alkalischen Reaktion des Blutes und folglich auch in die Ausscheidung von Säure und Alkali im Harn einzudringen, ist zweifellos Henderson (31). In einer Reihe von kritischen und experimentellen Arbeiten ist er zu Resultaten gekommen, die wir hier zusammenfassen können: Die Regulation der Reaktion des Blutes ist die Wirkung von zwei Faktoren: der Ausscheidung von Säuren vermittelst der Nieren, welches ein fast reiner physiko-chemischer Vorgang ist und der Ausscheidung des Ammoniaks, welche eine äquivalente Menge der fixen Alkalien des Blutes erspart. Die Gesamttwenge der ausgeschiedenen Säuren wird daher aus der Acidität des Harnes bestimmt, vermehrt um die Menge des ausgeschiedenen Ammoniaks.

Diese beiden Faktoren sind, nach dem Verfasser, vollständig verschieden, chemisch unabhängig und daher rein additive Phänomene. Im allgemeinen haben beide dieselbe Grösse, aber die Säureausscheidung ist an die Ausseheidung der Phosphate gebunden, während die des Ammoniaks von einer selektiven exkretorischen Tätigkeit der Niere abhängt, durch welche das Ammoniak im Harn quantitativ andere Basen des Blutes, je nach Bedürfnis ersetzt.

Nach einer etwas anderen Richtung hin und mit einer verschiedenen Methode hat Beccari (7) einen bemerkenswerten und entscheidenden Beitrag zur vorliegenden Frage gelicfert. Er hat vor allem seine Untersuchungen 
auf den Harn der reinen PHanzenfresser (Rinder, Pferde) gerichtet, indem er bei denselben die Ammoniakausscheidung mit genaueren analytischcn Methoden ausführte.

Er hat anf diese Weise eine wichtige Lücke ausgefüllt, weil wir für den vorliegenden Fall nur spärliche und fragmentarische Angaben besitzen, und ausserdem, wegen der unvollkommenen Untersuchungsmethoden, zweifelhafte. Die Ammoniakmenge, welche in ganz frisehem Harn von grossen Phanzenfressern mit normaler Nabrung enthalten ist, ist minimal und schwankt zwischen 10-25 mg pro 100, was in Durchschnitt $0,084 \%$ des (iesautstickstoffes beim Plerde und $0,178 \%$ beim Hunde ansmacht. Der Harn von solchen Tieren (mit Hafer und Heu gefüttert) hat eine stark alkalische Reaktion, die 5-8 $\mathrm{g} \mathrm{NaOH}$ pro Liter entspricht.

Bei denselben Tieren enthält das Blut Ammoniakmengen, welche kaum geringer als die bei den Fleischfressern gefundenen sind; dies ist besonders beim Hund der Fall, bei welchem man häufig die höchsten Zahlen für Ammoniak im Harn findet. Aus dieser Tatsache lässt sich sehliessen, dass die geringere Ausseheidung von Ammoniak bei den reinen Pflanzenfressern nicht vou einer vollständigeren Umwandlung der Ammoniakbestandteile in Harnstoff abhängt, sondern von den Beziehungen, welche zwischen den äquivalenten Säuren und Basen im zirkulierenden Blute bestehen. Beccari nimmt an, dass unter physiologischen Bedingungen sowohl die harnstoffbildende wie die antagonisierende Wirkung der Säure des Ammoniaks in engster Abhängigkeit von den Beziehungen sein müssen, welche zwischen den H-Ionon und den OH-Ionen des Plasmas und den Flüssigkeiten, welche die anatomischen Elemente umgeben, die an diesen Vorgängen beteiligt sind, hestehen.

Der Übertritt von Ammoniak in den Ham in kleineren oder grösseren Mengen hängt nicht von der Proportion des Ammoniaks im Blute ab, welche nicht variiert. Er scheint auch nicht in Verbindung mit dem grösseren oder kleineren Reichtum an festen Alkali des Plasmas zu steben, sondern er hänğt sicherlich von den Beziehungen zwischen H-Ionen und OH-Ionch dos Plasmas ab, welche meistens die Reaktion des Harnes bestimmen.

Um diesen P'unkt des Problems zu erforschen, hat Beccari versucht mit der grössten Genauigkeit die Summe der äquivalenten Säuren oder Basen des Harns in Beziehung zur Ammoniakausscheidung unter verschiedenen Bedingungen zu bestimmen. Lis gab aber keine sichere Methode für solche Untersuchungen. Wie bekannt, ist die einzige, welche theoretisch diescm Zwecke entsprechen würde, diejenige von Maly und Hofmann, welche unglücklicherweise Fehlerquellen aufweist, die sie unbrauchbar macht.

Beccari (8) ist es gelungen sie derart zu modifizieren, dass sie genau und felılerfrei geworden ist. Wie bekannt, wird die Reaktion des Harns, welche gewöhnlich sauer ist, durch saure Salze (Phosphate, harnsaure Salze) bestimmt, welche keine genaue Bestimmung mit den direkten Indikatoren 
gestatten. Bei der Methode von Maly werden diese Radikale (Phosphate usw.) durch BaCl gefält und die Lösungen mit Natronlauge titriert und im Filtrat der Überschuss der Natronlauge zurücktitriert, welcher nicht dazu gedient hat die H-Ionen der vorhandenen Phosphorsäure zu sättigen. Aber bei solchen Reaktionen treten wegen der basischen Bayrumphosphate und wegen der Gegenwart von $\mathrm{CO}_{2}$ beständig Fehler auf, welche die Methode fiïr genaue Versuche unbrauchbar macht. Beccari hat diese Fehlerquellen heseitigt, indem er zuerst die Kohlensäure aus dem Harn entfernte uni dann die Phosphate (und harnsauren Kalke) vermittelst Baryum als normale Salze fällte, mit einer Modifikation, welche die Methode vollkommen zuverlässig gestaltet.

Auf diese Weise konnte er mit absoluter Genaugkeit die wirklichen imol quantitativen Vorhältnisse untersuchen, welche zwischen der Summe der Säureäquivalente einerseits und dem Ammoniak und dor Plosphorsäure andererseits eintreten.

Da er beim Menschen die Ausscheidung soleher Stoffe zu den verschiedenen Tagesstunden, vor und nach den Mahlzeiten untersuchte, hat er den Parallelismus zwischen der Acidität des Harnes und der Ausscheidung des Ammoniaks und des Phosphorsäureanhydrids und der Funktion der Sättigung les Ammoniaks in bezug auf die Phosphorsäure genau bestimmen können und inwielern dieses das Basenäquivalent darstellt, welches vom Organis. mus bei der partiellen Sättigung der Phosphorsäure, die zur Säureausscheidung bestimmt ist, benötigt wird. Indem er so dem Ammoniak des Harns diese Bedeutung zuschrieb, folgt daraus, dass die gesamte Sänreausseheidung des Organismus aus der Summe der Säureäquiva lente (nach der Methode von Maly-Beceari frei gemacht und bestimmt) und den Aquivalenten des Ammoniaks (äquivalente Säuren mit $\mathrm{NH}_{3}$ gesättigt) berechnet wird.

In einer anderen Versuchsreihe (8) hat derselbe Forscher die Modifikationen untersucht, welche die Ausscheidung des Ammoniaks und der Phosphorsäure in Harn beim Menschen und bei Tieren erleidet, wenn die Reaktion des Harnes modifiziert wird.

Beim Plerde hat er, indem er aus dem Umstande Nutzen rog, dass durch die reine Hafernahrung die Reaktion des Harnes sauer wird, wie beim Menschen und beim Hund, zwei interessante Tatsachen zeigen können, und zwar: dass in dem Augenblick, in welchem die alkalische Reaktion sauer wird, das Ammoniak plötzlich zunimmt und gleichzeitig (eine noch interessantere Tatsache) im Ham die Phosphorsäure auftritt, welche im Harn mit alkalischer Reaktion vollkommen fehlt.

Beim Menschen und beim Hunde hat er mit grosser Genauigkeit den Einfluss beobachtet, welchen die eingeführten Alkalien auf die Ausscheidung des Ammoniaks und der Phosphorsäure in Beziehnng zur Harnreaktion hat.

Der Gegenstand ist schon von Salkowski und Munk, von Coranda 
und neuerdings von Haskins, Kowalewski und Salaskin behandelt worden, aber die quantitativen Verhältnisse zwischen den Äquivalenten in den verschiedenen Phasen der Sekretion wurden nicht untersucht. Obwohl die erhaltenen Resultate nicht zu einer fast mathematischen Definition der Verhältnisse zwischen Äquivalenten der Säuren und des Ammoniaks genügen, hat Beccari jedoch beim Hunde Bedingungen erreichen können, welche denjenigen der Pflanzenfresser älnlich waren, indem er mit der Nahrung beträchtliche Mengen von Natriumacetat darreichte, nämlich einen Grad der Alkalinität des Harnes, welcher demjenigen alınlich war, den man beim Pferd mit Heunahrung während einer ziemlich langen Periode antreffen kann; parallel hierzu erreicht die Abnahme des Ammoniaks im Harn die untersten beim Pferd beobachteten Grenzen, die beim Hunde nie erreicht worden sind $(0,0034 \mathrm{~g}$ pro 100 , d. L. $0,065 \%$ des Gesamtstickstoffes). Das beweist, dass wenn auch bei den Fleischfressern die Ammoniakausscheidung Grenzen erreichen kann, welche bei den Pflanzenfressern normal sind, eine solche Wirkung von dem Überfluss fixer Alkalien herrührt, die nur vorübergehend erhalten werden kann, welche aber jedenfalls beweist, dass der regulatorische Mechanismus der Ammoniakausscheidung von denselben Faktorcn abhängt ma bei den verschiedenen Tierarten denselben Gesetzen folgt.

1)iese Faktoren sind nach Beccari von den Gleichgewichtsbedingungen zwischen $H$-Ionen und $O H \cdot I o n e n$ abhängig, welche im Gebiete des Nierenepithels herrschen.

Eine neue wichtige Funktion, ausser der von Beccari nachgewiesenen, wird jetzt dem Ammoniak auf Grund von Untersuchnngen von Grafe, von Taylor und I. Ringer t1. a. zugeschriehen, deren Resultate nicht iuhereinstimmen.

Einige Forscher hatten schon beobachtet, dass bei grossen Pflanzenfressern die Darreichung von Asparagin und Ammoniaksalzen einen günstigen Einfluss auf den Eiweissstoff wechsel hatte, aber die Resultate sind ron anderen Forschem ihrer Beweiskraft beraubt worden (53). So haben Embren und Schmitz (14) mit Beweisen und Tatsachen an der Leber, die Glykogen enthielt und überlebend der künstlichen Zirkulation unterworfen wurde, festgestellt, dass sich in derselben aus Kohlehydraten und Ammoniak Alanin bildet.

Aber die bemerkenswerte Arbeit von Grafe und Schlaepfer (24) lenkte die Aufmerksamkeit wieder auf diese Frage und lieferte hierzu vermittelst genauer Versuche an Humden, welche vorher 8-22 Tage hungerten, einen neuen wertvollen Beitrag. Dieselben wurden mit stickstofffreier Weizenstärke, Rohrzucker, etwas Butter, einigen 'Tropfen Fleischextrakt, der annähernd eiweissfrei war, etwas Suppe, $3 \mathrm{~g}$ Salz und $500 \mathrm{~g}$ Wasser gefüttert; hier\%u wurde in der zweiten Versuchsperiode Ammoniumcitrat linzugefügt. Während dieser zweiten Periode beobachteten sie eine starke Stickstoffretention. Aucl: mit grossen Mengen von Ammoniumcitrat gelang es die Tiere längere Zeit 
im Stickstoftgleichgewicht zu halten. Gleichzeitig nahm das Körpergewicht zu, während mit derselben reichlichen Ernährung ohne Zusatz von Ammoniaksalzen eine bemerkenswerte Gewichtsabnahme beobachtet wurde. Dieselben Resultate erzielten sie beim Menschen (25).

Grafe nimmt an, dass es sich um einen synthetischen Vorgang handelt, welcher die Bildung von Eiweiss aus Ammoniak und Kohlehydraten veranlasst. Die Untersuchungen von Knoop (42) und Embden (14), welche die Möglichkeit einer ähnlichen Synthese gezeigt haben, würden zugunsten der erwähnten Erklärung sprechen.

Fast gleichzeitig kam Abderhalden (1), welcher Hunde mit Fetten, Kohlehydraten und Ammoniumacetaten füiterte, zu demselben Scbluss wie Grafe.

Jedoch erkannte er in einer späteren Arbeit mit Hirsch (2), dass eine andere Stickstoffquelle, das Erepton (gänzlich abgebautes Fleisch) und das Ammoniumacetat sich ganz verschieden bei jungen Hunden verhalten. Während die Hunde mit Erepton eine Gewichtszunahme zeigten, nahmen sie bei Einführung von Gelatine und Ammoniumacetat an Gewicht ab. Übrigens haben auch Taylor und Ringer (67) bei Hunden und bei einem Menschen, welchen sie per os zwei Tage hintereinander $11,60 \mathrm{~g} \mathrm{Ammoniumacetat} \mathrm{gegeben}$ hatten, eine Ammoniakretention beobachtet; jene Forscher nehmen an, dass sich Aminosäuren bilden, welche zur Bildung und zur Ersparnis von Eiweiss im Körper dienen.

Wenn Underhill und S. Goldschmidt $(69,70,72)$ gesehen haben, dass nach Einführung von Ammoniumchlorid und Ammoniumsulfat das Ammoniak und der Stickstoff zunehmen, so muss man das nach Grafe den angewandten Dosen zuschreiben, während auch beim Menschen das Ammoniumchlorid eine deutliche Stickstoffretention hervorruft, wemn es nicht in giftigen Dosen gegeben wird.

Henriquez und Andersen (35), welchen es nicht gelungen war eine andauernde Stickstoffretention im Organismus vermittelst andauernder intravenöser Injektion von Zucker und Ammoniumacetat hervorzurufen, erklärten die von Grafe erhaltene Retention als den Ausdruck der bakteriellen Tätigkeit des Darmes. Diese Hypothese ist schon von Peschek (53) aufgestellt worden, nach welchem auf die intravenöse Injektion von Ammoniumsalzen Zunahme des Stickstoffverbrauches folgt.

In späteren Arbeiten hat Peschek $(54,55,56)$ Tatsachen von bemerkenswerter Bedeutung gefunden. Einerseits haben die Ammoniumsalze von verschiedenen organischen Säuren dieselbe Wirkung auf die Stickstoffretention; während Ammoniumacetat bemerkenswerte Retention erzeugt, fehlt dieselbe bei Ammoniumlactat. Andererseits hat derselbe Forscher gezeigt, dass auch der Nahrung zugesetztes Natriumacetat die Eigenschaft hat, eine Einschränkung der Stickstoffausscheidung zu bewirken; ähnliche Wirkungen haben 
das zitronensaure und milchsaure Natrium und das Magnesiumacetat. Auf Grund dieser Tatsachen nimmt Peschek an, dass die Verminderung der Stickstoffausscheidung nicht von der Komponente "Ammoniak" herrührt, sondern von dem indirekten Einfluss auf den Stickstoffstoffwechsel gewisser Salze (Essigsäure usw.) als solcher, da die entsprechenden freien Säuren keine analoge Wirkung auf diesen Stoffwechsel besitzen.

Auch Abderhalden kam in einer neueren Arbeit (3), welche eine Jange Versuclısreihe über den Einfluss der Ammonsalze, des Harnstoffs, Natriumacetats auf das Stickstoffgleichgewicht enthält, auf diese Frage zurück. Seine Resultate führen, ausser dass sie den oben beschriebenen Einfluss des Natriumacetats auf den Stickstoffstoffwechsel bestätigen, zu dem Schluss, dass wenn einerseits, während der Einführung der Ammonsalze eine Stickstoffretention eintritt, andererseits in der Periode, welche auf diese Einführung folgt, eine Stickstoffausscheidung zu beobachten ist, welche die vorangehende Retention zu kompensieren scheint.

Ein entscheidender Beweis über den Einfluss der Ammonsalze über den Eiweissverbrauch des Organismus scheint, unter Leitung des oben zitierten Grafe, von Gessler (23) geliefert worden zu sein, welcher bei hungernden Hunden gezeigt hat, dass die Einführung von Ammoniaksalzen absolut nicht die Schwefelausscheidung modifiziert, welche vom Eiweissverbrauch des Organismus herrührt. Die Verminderung der Stickstoffausscheidung würde folglich von der Retention des Ammoniakstickstoffes herrühren.

Es ist für den Augenblick nicht möglich zu entscheiden in welcher Form dieser Stickstoff zurückgehalten wird, oder ob er auf irgend eine Weise aur. genützt werden kann. Immerhin können wir nicht unterlassen auf die letæten Beobachtungen von E. Salkowski $(60,61)$ an Wiederkäuern hinzuweisen, wonach bei diesen Tieren bei einer Nahrung, die frei von verdaulichem Stickstoff ist und Zucker, Stärke und Harnstoff enthält, täglich $10-20 \mathrm{~g}$ Eiweiss erzengt werden. Beim kastrierten Tier, welches ausschliesslich mit Stroh, d. h. mit einer der vorhergehenden entsprechenden Nahrung ernährt wurde, gab es in 155 Tagen eine Körpergewichtszunahme von $30-39 \mathrm{~kg}$, es bildeten sich also $6,336 \mathrm{~kg}$ Fleisch. Der Schwefel entstammte den Sulfaten. 